\title{
A Network Approach to Parental Burnout
}

M. Annelise Blanchard ${ }^{1}$, Isabelle Roskam ${ }^{1}$, Moïra Mikolajczak ${ }^{1}$, and Alexandre Heeren ${ }^{1,2}$

${ }^{1}$ Psychological Sciences Research Institute, UCLouvain, Belgium

${ }^{2}$ Institute of Neuroscience, UCLouvain, Belgium

\section{Accepted for publication in Child Abuse \& Neglect}

This is a preprint (version Nov 17, 2020) of a manuscript that has been peer-reviewed and accepted for publication in Child Abuse \& Neglect. This paper is not the copy of record and may not exactly replicate the final, authoritative version of the article as published in Child Abuse \& Neglect. The final article will be available, upon publication, via its DOI. Analysis code and data can be found in the accompanying OSF project: https://osf.io/dxtgz/

\section{Author Note}

M. Annelise Blanchard (iD https://orcid.org/0000-0002-9605-7022

Isabelle Roskam (D) https://orcid.org/0000-0002-1449-1133

Moïra Mikolajczak (D) https://orcid.org/0000-0002-7333-1578

Alexandre Heeren (iD https://orcid.org/0000-0003-0553-6149

The data analysis for this study was preregistered on the Open Science Framework (https://osf.io/2r6bv). The anonymized summed data, as well as the R code used for analyses, are also both available on the Open Science Framework (https://osf.io/dxtgz/).

M. Annelise Blanchard received the "2020 Student Development Award" of the Stress and Anxiety Research Society-i.e., an annual competition for the best paper on stress, anxiety, and coping in theory, practice or research - for a preprint version of this manuscript.

Moïra Mikolajczak. and Isabelle Roskam have founded the "Training Institute for Parental Burnout," which delivers training on parental burnout to professionals. The institute did not participate in the funding of this study nor did it influence the process or the results in any manner. The other authors have no known conflict of interest to disclose. 
All the authors were supported by a Coordinated Research Grant ("BParent") from the French Community of Belgium (ARC Grant 19/24-100). M. Annelise Blanchard (as FRSFNRS research follow) and Alexandre Heeren (as FRS-FNRS research associate) are also supported by the FRS-FNRS Belgian National Science Foundation. These funds did not exert any influence or censorship of any kind on the present work.

Correspondence concerning this article should be addressed to M. Annelise Blanchard. Psychological Sciences Research Institute, Université Catholique de Louvain, Place du Cardinal Mercier, 10, B-1348, Louvain-la-Neuve, Belgium. E-mail:

marie.blanchard@uclouvain.be 


\begin{abstract}
Background: The use of network analyses in psychology has increasingly gained traction in the last few years. A network perspective views psychological constructs as dynamic systems of interacting elements.
\end{abstract}

Objective: We present the first study to apply network analyses to examine how the hallmark features of parental burnout - i.e., exhaustion related to the parental role, emotional distancing from children, and a sense of ineffectiveness in the parental role — interact with one another and with maladaptive behaviors related to the partner and the child(ren), when these variables are conceptualized as a network system.

Participants and setting: In a preregistered fashion, we reanalyzed the data from a Frenchspeaking sample $(n=1551$; previously published in Mikolajczak, Brianda, Avalosse, \& Roskam, 2018), focusing on seven specific variables: the three hallmark parental burnout features, partner conflict, partner estrangement, neglectful behavior toward children, and violent behavior toward children.

Methods: We computed two types of network models, a graphical Gaussian model to examine network structure, potential communities, and influential nodes, and a directed acyclic graph to examine the probabilistic dependencies among the different variables.

Results: Both network models pointed to emotional distance as an especially potent mechanism in activating all other nodes.

Conclusions: These results suggest emotional distance as critical to the maintenance of the parental burnout network and a prime candidate for future interventions, while affirming that network analysis can successfully expose the structure and relationship of variables related to parental burnout and its consequences related to the partner and the child(ren).

Keywords: Parental burnout, network analysis, neglect, violence, parental conflicts, directed acyclic graph 


\section{A Network Approach to Parental Burnout}

A growing number of fields, including ecology, biology, and physics, have within the last decade embraced a network perspective, in line with both a growing interest and an increasing computational capability to model the complexity of systems (Barabási, 2012). Psychology subfields, including personality (e.g., Cramer et al., 2012) and psychopathology research (for systematic reviews, see Contreras et al., 2019; Robinaugh et al., 2019), have also recently embraced a network perspective, viewing psychological constructs as dynamic systems of interacting elements. Instead of investigating a construct as if it were a unitary phenomenon measured by multiple variables, a network approach examines and visualizes how the variables themselves interact with one another (for a detailed and accessible introduction to network analysis, readers can refer to the reviews by Borsboom, 2017; Borsboom \& Cramer, 2013; Contreras et al., 2019; McNally, 2016; Robinaugh et al., 2019).

Exploring the structure and topology of a network of psychological variables can thus grant insight into the organization and mechanisms of psychological constructs. For example, nodes that are central to a network (and so especially connected to other nodes) work to maintain the network system, since once activated they can quickly spread that activation (Borgatti, 2005; Costantini et al., 2015). Within network perspectives of psychopathology, central nodes have been theoretically (Borsboom \& Cramer, 2013) and empirically (Elliott et al., 2019; Papini et al., 2020) linked with the prognosis of a disorder. Assessing network structure also allows researchers to investigate whether nodes cluster together into communities and whether specific nodes act as bridges to connect these potential communities (Jones et al., 2019). For psychopathology networks, understanding community structures and identifying potential bridge nodes is useful when examining comorbidity, but also more broadly to understand how specific symptoms might be crucial to the network system (McNally, 2016). Bringing a network perspective to psychology research thus allows 
researchers to identify key variables that work to galvanize other variables or that connect multiple constructs.

A psychological phenomenon that is optimal for a network perspective is parental burnout. Parental burnout occurs when parents chronically lack the parenting resources needed to cope with their specific parenting-related stressors and involves three hallmark features, namely: 1) an overwhelming exhaustion related to the parental role, 2) an emotional distancing from one's children, and 3) a sense of ineffectiveness and loss of accomplishment in the parental role (Mikolajczak \& Roskam, 2018; Roskam et al., 2017). Although epidemiological studies have not yet been published, recent assessments estimate that $5 \%$ of parents experience at least two of these three hallmark features daily (Roskam et al., 2018). However, although the concept of parents experiencing burnout has previously been investigated with parents of ill children (e.g., Lindström et al., 2011), it has only recently been examined in general populations of parents (Mikolajczak et al., 2020; Roskam et al., 2017). Still, the notion of parental burnout has gained traction (e.g., Cheng et al., 2020; Sorkkila \& Aunola, 2020) and increasingly widespread recognition (e.g., Griffith, 2020).

Parental burnout presents itself as a prime candidate for a network analysis for two important reasons (Blanchard \& Heeren, 2020). First, uncertainty persists regarding how the three hallmark features of parental burnout interact together. Indeed, in most of the previous parental burnout studies that have been published so far, researchers have tallied items of the three features to achieve a sum-score as an index of parental burnout (Blanchard \& Heeren, 2020). This is unfortunate as one may easily envision how these features might interact with one another (e.g., a sense of ineffectiveness and loss of accomplishment in one's parental role may foster emotional distancing from one's children, and vice versa; an overwhelming exhaustion related to one's parental role may cultivate a sense of ineffectiveness and loss of 
accomplishment in one's parental role, and vice versa). A critical step would thus be to elucidate the functional relations among these features.

Second, parental burnout can easily be conceptualized as a dynamic system of interacting variables, with the three hallmark features of parental burnout impacting the entire family system (Blanchard \& Heeren, 2020). Indeed, parental burnout impacts more than just the affected parent. This is because one parent feeling burned out is linked with family-wide dysfunction, including within the couple's relationship (e.g., conflict and estrangement) but also including severe negative consequences for the children, such as neglect and violence (Mikolajczak, Brianda, et al., 2018). Research investigating the effects of parental burnout is still in its beginning stages, but what literature does exist supports that parental burnout is linked with harmful parenting behaviors (Hansotte et al., 2019; Mikolajczak et al., 2019). These findings are not surprising, as previous research has found that with an increase in parental stress comes an increase in abusive behaviors (Berger et al., 2011; Curtis et al., 2000). However, as of yet no studies have untangled how the main features of parental burnout impact each other and dysfunctional family functioning, and vice versa. We thus propose a network analysis approach, to investigate how the hallmark features of parental burnout interact with each other and with variables implicated in family dysfunction namely, a couple's relationship with each other (including conflict and estrangement) and their behavior toward their children (including neglect and abuse).

In this project, we have three primary goals. First, we endeavor to test whether the hallmark features of parental burnout-i.e., an overwhelming exhaustion related to the parental role, an emotional distancing from the child(ren), and a sense of ineffectiveness and lack of accomplishment in the parental role-cohere as a network system. Relatedly, we aim to elucidate the pairwise connections between these features and maladaptive behaviors relating to the partner (i.e., marital conflict, partner estrangement) and the children (neglect 
and violence toward children). Of critical interest will be the computation of centrality metrics that disclose the potential most influential nodes that maintain the network system (Borgatti, 2005; Costantini et al., 2015).

Second, we will test whether these nodes cohere as a unitary network of interacting elements or whether they constitute distinct communities (or subnetworks) of nodes serving different functions. And, if we find evidence for distinct communities, we will investigate whether certain nodes function as "bridges," i.e., processes that connect or are shared by communities. These analyses will offer viable heuristics to identify especially potent nodes that may kindle mutually reinforcing interactions between the features of parental burnout and maladaptive behaviors toward the family.

Finally, we will use Bayesian methods to compute a directed acyclic graph (DAG) to estimate a directed, potentially causal model of the interplay among the key features of parental burnout and maladaptive behaviors toward the partner and children. A DAG is a directed network whereby each edge has an arrow tip on one end, signifying the direction of prediction (and possibly causation), or, more conservatively, the direction of probabilistic dependency (e.g., Heeren et al., 2020; McNally, 2016; McNally et al., 2017; Pearl et al., 2016; Scutari, 2010). These complementary approaches can provide both a novel perspective and potential new hypotheses about plausible causal connections that merit more rigorous followup research. All of these computations will be performed as a re-analysis of an existing dataset (Mikolajczak, Brianda, et al., 2018) that includes questionnaires relating to parental burnout, animosity between the parents, and child maltreatment.

\section{Method}

\section{Preregistration}

As this is an exploratory study using secondary data, we followed the guidelines of Weston, Ritchie, Rohrer and Przybylski (2019) and preregistered the analysis plan, 
methodology, and prior knowledge regarding the dataset at https://osf.io/32vhg/. De-identified data as well as R code are publicly available via the Open Science Framework and can be accessed at https://osf.io/dxtgz/. We did not deviate from our preregistration.

\section{Participants}

We reanalyzed data from a previous study (Mikolajczak et al., 2018), using only the data from individual participants who had completed full questionnaires regarding their own parental burnout, their own partner-related behaviors, and their own violent and neglectful behaviors toward their own children. This yielded a sample of 1551 French-speaking participants (75.8\% women). Participants were recruited from the general community (i.e., unselected sample) via websites, social media, schools, and pediatricians inviting people to participate to an online survey. All participants were parents with at least one child living at home, and participants were rewarded with the opportunity to participate in a lottery. Further details about the data collection can be found in the original article (Mikolajczak, Brianda, et al., 2018). The initial study was approved by the institutional review board of the Psychological Sciences Research Institute (UCLouvain, Belgium) and conducted according to the Declaration of Helsinki. Participants' characteristics appear in Table 1.

\section{Measures}

\section{Parental Burnout}

We used the Parental Burnout Inventory (PBI), a 22-item self-report questionnaire designed to measure the severity of parental burnout (Roskam et al., 2017). It includes three subscales that map directly onto the three hallmark features of parental burnout: a) eight items measuring emotional exhaustion (e.g., "Ifeel emotionally drained by my parental role”); b) eight items measuring emotional distancing (e.g., "I sometimes feel as though I am taking care of my children on autopilot"); and c) six items measuring parental accomplishment and efficacy (e.g., "I accomplish many worthwhile things as a parent”). Participants rated each 
item on a 7-point Likert-type scale, from 0 (never) to 6 (every day). Items denoting the absence of the feature of interest (e.g., "I look after my children's problems very effectively") were reverse-scored prior to computing each subscale's total by summing the relevant item scores.

Scores on the PBI have excellent internal consistencies and good validity (Roskam et al., 2017). In the present dataset, the internal reliability of PBI was high, with a Cronbach's alpha of .93 for the global scale score (.94 for the emotional exhaustion, .88 for the emotional distancing, and .86 parental accomplishment and efficacy). Given our interest in distinguishing between the three features, we computed separate scores for the three subscales. For each subscale, higher scores indicate greater endorsement of that feature. The scores for the emotional exhaustion subscale could range from 0 to 48 ; from 0 to 48 for the emotional distance subscale; and from 0 to 36 for the inefficacy subscale.

\section{Conflicts with Partner}

Conflicts were measured with two self-report items as reported in Mikolajczak, Brianda, et al. (2018): "How often do you quarrel with your partner?" and "How often do you quarrel with your partner in front of your child(ren)? " Participants rated items on a 7point Likert-type scale, from 1 (never) to 7 (every day). For each participant, we computed a sum score: scores could range from 2 to 14. In the present dataset, the internal reliability of this scale was high, with a Cronbach's alpha of .85 .

\section{Partner Estrangement}

Partner estrangement was assessed with a five-item self-report questionnaire, as reported in in Mikolajczak, Brianda, et al. (2018). These items include: "I sometimes fantasize about someone other than my partner," "I am sometimes unfaithful to my partner," "We have separate rooms or I go to sleep elsewhere, " I sometimes think of leaving my partner, " and "I threaten my partner with leaving." Participants rated each item on 8-point Likert-type scale 
ranging from 1 (never) to 8 (several times a day). For each participant, we computed a sum score: scores could range from 5 to 40 . In the present dataset, the internal reliability of this scale was adequate, with a Cronbach's alpha of .73 .

\section{Neglectful Behaviors Toward Children}

Neglectful behavior toward children was assessed via a 17-item parent-reported questionnaire (for details on how this questionnaire was derived, see Mikolajczak, Brianda, et al., 2018). Sample items include: "I sometimes neglect my child when s/he is sad, frightened or distraught" and "I sometimes don't take my child to the doctor when I think it would be a good idea." The parent rated each item on an 8-point Likert-type scale ranging from 1 (never) to 8 (several times a day). In the present dataset, the internal reliability of this scale was good, with a Cronbach's alpha of .82. Accordingly, we relied on a sum score, and scores could range from 17 to 136 .

\section{Violent Behaviors Toward Children}

Violent behavior toward children was assessed with a 15-item parent-reported questionnaire (for details on how this questionnaire was derived, see Mikolajczak, Brianda, et al., 2018). Sample items include: "I sometimes hurt my child with a belt, a hairbrush, a stick or some other object" and "I sometimes tell my child that I will abandon him/her if s/he is not good." Items were rated with the same scale as used for the neglectful behaviors scale. The internal reliability of this scale was good, with a Cronbach's alpha of .82. We computed a sum score for each participant, and scores could range from 15 to 120 .

\section{Network analyses}

\section{Data preparation}

Following recent guidelines in the application of network analysis in clinical psychology (e.g., Epskamp \& Fried, 2018), we applied the nonparanormal transformation via the R package huge (Jiang et al., 2019). This transformation ensures that the data are 
multivariate normal and thus meet relevant assumptions for use with the estimated network analyses (Epskamp et al., 2018).

To ensure that none of our variables overlapped conceptually, we implemented a datadriven method to identify potentially redundant nodes among our seven variables (as reported in Bernstein et al., 2019). We first confirmed that our correlation matrix was positive definite, reflecting that nodes were not linear combinations of other nodes). We then searched for potential pairs of redundant nodes; that is, nodes that are highly inter-correlated $(r>0.50)$ and that correlate to the same degree with other variables (i.e., $>75 \%$ of correlations with other variables did not significantly differ for a given pair). To do so, we implemented the Hittner method for comparing dependent correlations (Hittner et al., 2003) via the goldbricker function of the R package networktools (Jones, 2018). This method did not identify any redundant variables.

\section{Graphical LASSO Network}

We used a graphical Gaussian model (GGM) to estimate the undirected network. In this network, edges signify conditional independence relationships between nodes, controlling for the effects of all other nodes (Epskamp \& Fried, 2018). We present GGMs that were regularized via the graphical LASSO (Least Absolute Shrinkage and Selection Operator) algorithm, which served two primary functions (Friedman et al., 2011). First, it computed regularized partial correlations between pairs of nodes, thereby eliminating spurious associations (edges) attributable to the influence of other nodes in the network. Second, it shrunk trivially small associations to zero, thereby removing potentially "false positive" edges from the graph and producing a sparse graph comprising only the strongest edges. We used the R package qgraph (Epskamp et al., 2012), which automatically implements the graphical LASSO regularization in combination with the Extended Bayesian Information Criterion (EBIC) model selection (Foygel \& Drton, 2011). This procedure estimates 100 models with 
varying degrees of sparsity; a final model is selected according to the lowest EBIC value, given a specific hyperparameter gamma $(\gamma)$ which controls the trade-off between including false-positive edges and removing true edges. The hyperparameter $\gamma$ is usually set between 0 (favoring a model with more edges) and 0.5 (favoring a simpler model with fewer edges). Following recommendations based on stimulation studies (for details, see Epskamp \& Fried, 2018), we set $\gamma$ to 0.5 to be confident that our edges are genuine. To estimate the stability of edges, we bootstrapped the confidence regions of the edge weights with 1,000 bootstrapped samples using the R package bootnet (Epskamp et al., 2018).

To quantify the importance of each node in the resulting graphical LASSO network, we computed expected influence centrality (Robinaugh et al., 2016). The expected influence of a node is the sum of the edge weights incident on a given node, including positive and negative values. Higher expected influence values indicate greater centrality and so greater importance in the network. The plot depicts the raw expected influence values for each node.

Finally, we tested whether the nodes denoting the key features of parental burnout and those denoting maladaptive behaviors relating to the partner and the children cohere as one network or as multiple subnetworks ("communities"). Nodes within a community are more strongly interconnected than they are with nodes outside that community. Following prior network research (e.g., Heeren \& McNally, 2018; Robinaugh et al., 2014), we implemented the spin glass algorithm (Reichardt \& Bornholdt, 2006), a modularity-based community detection procedure suitable for uncovering the structure of relatively small networks with negative edge values (e.g., Traag \& Bruggeman, 2009). We used the spinglass.community function $(\gamma=1$, start temperature $=1$, stop temperature $=.01$, cooling factor $=.99$, spins $=7)$ of the R package igraph (Csardi \& Nepusz, 2006; for more information, see Reichardt \& Bornholdt, 2006; Traag \& Bruggeman, 2009; Yang et al., 2016). Following previous studies (e.g., Everaert \& Joormann, 2019; Heeren, Jones, et al., 2018; Heeren et al., 2020), we also 
identified important nodes that serve as bridges between communities by computing the bridge expected influence index via the bridge function of the R package networktools (Jones, 2018). Nodes with high bridge expected influence values are especially likely to activate nearby communities. Bridge expected influence is the sum of the edge weights connecting a given node to all nodes in the other community or communities (Jones et al., 2019). The plot depicts the raw bridge expected influence values for each node.

\section{Directed Acyclic Graph (DAG)}

We then computed a DAG to estimate the directed structure of the system (Pearl et al., 2016). We used a Bayesian hill-climbing algorithm, implemented via the R package bnlearn (Scutari, 2010; Scutari \& Denis, 2015). As implemented by bnlearn, the bootstrap function computes the structural aspects of the network model by adding edges, removing them, and reversing their direction to ultimately optimize the goodness-of-fit target score (i.e., the Bayesian Information Criterion). This involves an iterative procedure of randomly restarting this process with different possible edges linking different node pairs, perturbing the system, and using 50 different random restarts to avoid local maxima. Following previous studies (Bernstein et al., 2017; Heeren et al., 2020; McNally et al., 2017), we performed 100 perturbations (i.e., attempts to insert, delete, or reverse an edge) for each restart. As this iterative procedure unfolds, the function returns the best fitting network based on this random restart/perturbation process.

To ensure the stability of the resultant DAG, we then bootstrapped 10,000 samples (with replacement), computed a network for each sample, and averaged across the resulting networks to produce a final network structure (e.g., Heeren et al., 2020; McNally et al., 2017), involving a two-step procedure. First, we determined how frequently a given edge appeared in the 10,000 bootstrapped networks. We then used the optimal cut-point method of Scutari and Nagarajan (2013) for retaining edges, which yields networks with both high sensitivity and 
high specificity. Second, we ascertained the direction of each surviving edge in the 10,000 bootstrapped networks. To do so, we followed Scutari and Nagarajan (2013)’s recommandations and only represented the direction of a surving edge in the final network if this edge pointed from node $\mathrm{X}$ to node $\mathrm{Y}$ in at least $51 \%$ of the 10,000 bootstrapped networks. In summary, we first determined the structure of the network (i.e., whether an edge is present or not), and then determined the direction of each surviving edge.

\section{Results}

Descriptive information regarding the seven variables (before nonparanormal transformation), including mean, standard deviation, range, skewness, and kurtosis, can be found in Table 2. Pearson product-moment correlations of nonparanormal-transformed variables are reported in Figure 1 (for a discussion of why we present Pearson correlations of transformed variables and not Spearman correlations of the raw data, see the supplementary materials).

\section{Graphical LASSO Network}

The graphical LASSO is represented in Figure 2. The edges represent regularized partial correlations between variables. All of the associations between variables are positive, as all of the edges are green (any negative edges would be represented in red). A few pairwise connections stand out. First, the largest edge weight is between emotional exhaustion and emotional distance $(r=.43)$. Second, whereas emotional exhaustion has direct connections with violent behaviors toward children $(r=.15)$ and conflict with the partner $(r=.13)$, emotional distance is strongly connected to neglectful behaviors toward children $(r=.36)$ and loss of parental accomplishment and efficacy to violent behaviors toward children $(r=.21)$. To estimate the certainty and precision of the edge weights, we bootstrapped confidence intervals for each of the edge weights (Epskamp et al., 2018) and also performed bootstrapped difference tests. Results support that the edges are stable, and that the strongest and weakest 
edges are significantly different from one another (see Figures S1 \& S2 in the supplementary materials, which can be found at https://osf.io/q56sn/).

Expected influence values are reported in Figure 2. Emotional distance, neglect towards children, and violence toward children had the highest expected influence values. To ensure the stability of these centrality estimates, we performed a person-dropping bootstrap procedure (Costenbader \& Valente, 2003), which confirmed that these expected influence values are highly stable (see Figure S3 in the supplementary materials). We also performed a bootstrapped different test, which revealed that the most central nodes (i.e., emotional distance, neglect, and violence) have significantly higher expected influence estimates than less central nodes (including the partner-related variables and the remaining parental burnout features; see Figure S4 in the supplementary materials).

Lastly, the spin glass algorithm detected three communities of nodes. The first community included emotional distance, emotional exhaustion, neglect, and violence; a second community solely encapsulated loss of parental accomplishment and efficacy; and a third community included partner-related behaviors (conflict and estrangement). Figure 3 shows the bridge expected influence values for all nodes, revealing that inefficacy has an especially high bridge expected influence value. We performed a person-dropping bootstrap, which indicated that bridge expected influence values were reasonably stable (see Figure S3 in the supplementary materials). A bootstrapped different test confirmed that inefficacy had a significantly higher bridge expected value than the remaining variables (see Figure S5 in the supplementary materials).

\section{Directed Acyclic Graph}

Figures 4A and 4B show directed acyclic graphs (DAGs) resulting from 10,000 bootstrapped samples. For both Figures $4 \mathrm{~A}$ and $4 \mathrm{~B}$, edges that are present in the graph were retained because their strength was greater than the optimal cut resulting from the method of 
Scutari and Nagarajan (2013). Figure 4A illustrates the importance of each edge to the overall network structure. Specifically, edge thickness reflects the change in the Bayesian Information Criterion (BIC; a relative measure of a model's goodness-of-fit) when that edge is removed from the network. Greater thickness thus signifies that an edge is more crucial to model fit (McNally, 2016). The edges most important to the network structure connect emotional distance to exhaustion (with a change in BIC of -338.29) and emotional distance to neglect (with a change in BIC of -167.37). The edges least important to the network structure, meanwhile, connect emotion exhaustion to partner conflict (with a change in BIC of -24.10) and emotional exhaustion to violence (with a change in BIC of -29.57). The change in BIC value for each edge can be seen in Table S1 in the supplementary materials.

In Figure 4B, edges signify directional probabilities: an edge is thicker if it points from one node to another in a greater proportion of the bootstrapped networks. The thickest edges connect emotional distance to violence (.82; i.e., this edge was pointing in that direction in 8,200 of the 10,000 bootstrapped networks and in the other direction in 1,800 of the 10,000 bootstrapped networks) and emotional exhaustion to violence (.81). The thinnest edges connect inefficacy to violence (.51) and emotional distance to exhaustion (.53). The exact directional probability for each edge in Figure 4B can be found in Table S1 in the supplementary materials.

Structurally, emotional distance arises at the top of the DAG, directly influencing the other parental burnout variables (emotional exhaustion and inefficacy), which then directly influence violence toward children, which in turn directly influences partner conflict and neglect toward children, which both directly influence partner estrangement.

\section{Preregistered Additional Analyses}

Recent commentators have argued that differential variability — that is, the phenomenon that variables have drastically different variances - may distort conclusions 
about node centrality (e.g., Fried, 2016; Terluin, de Boer, \& de Vet, 2016). We confirmed that differential variability did not influence the interpretation of the centrality indices (see supplementary materials).

Following previous studies in the field (e.g., Mikolajczak, Raes, Avalosse, \& Roskam, 2018), we also tested whether our results differed depending on the gender of the parent or the number of children. Using the Network Comparison Test (van Borkulo et al., 2017), we found no evidence of a difference between the networks' overall connectivity or structure between mothers compared to fathers, or parents of one, two, or three and up children (see

supplementary materials). Only one edge differed between parents of one child and parents of two children (Table S2): the network for parents with one child does not include an edge between neglect and partner estrangement, whereas the network for parents with two children does.

\section{Discussion}

In this study, we set out to elucidate how parental burnout features interact among themselves and with family-related variables, as well as whether any specific variables are especially central to the way parental burnout operates within a family system. To do so, we computed network analyses by using two distinct computational network approaches: a GGM and a DAG. Perhaps the most striking finding was the observation that GGM and DAG pointed to emotional distance as an especially potent variable at play. In both network models, our results imply that when emotional distance is activated, it drives the activation of the rest of the network. Additionally, both models indicated that the parental burnout variables are mainly associated with partner conflict and partner estrangement through the child maltreatment variables. This suggests that it is not parental burnout per se that is associated with marital issues, but the child maltreatment accompanying parental burnout that, in turn, drives marital conflicts. This is also apparent in the high centrality values of the nodes 
representing neglect and violence toward children, which confirm that these variables connect a lot of the other variables in the network.

In terms of community detection, it is interesting that loss of parental accomplishment and efficacy forms its own community, while the other two hallmark features of parental burnout, emotional distance and emotional exhaustion, cohere together in a community along with neglect and violence. Perhaps inefficacy is on its own because it relates to the skillsbased parenting evaluation of loss of parental accomplishment and efficacy, which is qualitatively different from the more affective components of emotional exhaustion and emotional distance. Inefficacy demonstrating a high bridge expected influence value suggests that it bridges the different communities together and therefore is a possible target deserving a careful audit during prevention and treatment. However, seeing as it is the only community made up of one node, it is only logical for inefficacy to have a high bridge expected influence, since any of its connections to other nodes count as inter-community connections. It is also of note that emotional distance and emotional exhaustion are categorized into the same community as neglect and violence toward children. This implies that these four nodes are more strongly connected to each other than they are to nodes outside their community. This means that to reduce neglect and violence toward children, it would be important to target emotional distance and emotional exhaustion in treatment efforts.

Even though the three parental burnout scales are categorized into separate communities in this study, previous research supports that these three scales together adequately assess parental burnout, and factor analyses support the use of three subscales (Roskam et al., 2017). Although the current study uses the PBI (Roskam et al., 2017) which was adapted from the job-related Maslach Burnout inventory (Maslach et al., 2010), a new scale has since been developed using an inductive approach. This scale, the Parental Burnout Assessment (PBA; Roskam, Brianda, \& Mikolajczak, 2018), encompasses four subscales: 
emotional exhaustion in one's parental role, emotional distancing from one's children, feelings of being fed up, and contrast with a previous period. This implies that parents lose pleasure and fulfillment as parents, instead of losing their parenting accomplishment and efficacy, thus bringing an emotional component to this dimension. A critical next step would thus be to conduct a network analysis using the PBA to test whether a similar community structure as found in this study which uses the three PBI subscales also appears with the four PBA scales.

Overall, these results suggest that emotional distance plays a key role in propagating activations to the other parental burnout nodes, as well as child maltreatment and partner conflict ones. This nuances the common conception of emotional exhaustion as the core dimension of burnout (Halbesleben \& Bowler, 2007); it has been conceptualized as such partly because it is the common variable that ties disparate definitions of burnout together (e.g., Demerouti et al., 2001; Maslach et al., 2010; Pines \& Aronson, 1988) and because several studies suggested that it was the first step of the burnout process (Lee \& Ashforth, 1993; Leiter, 1993; Leiter \& Maslach, 1988). Emotional exhaustion also appears influential for conceptions of burnout within the parenting sphere: a recently developed theoretical framework for parental burnout posits that parental burnout arises when there is a chronic lack of resources to meet parenting needs, leading to a depletion of energy and so emotional exhaustion (Mikolajczak \& Roskam, 2018). Yet in our results, emotional distance was more central in the GGM and more highly placed in the DAG than emotional exhaustion. A potential reason for this could be that this data was collected at one time-point, and in Mikolajczak and Roskam's (2018) framework, emotional exhaustion was specifically posited as a key variable in the development of parental burnout. As our study relies on a crosssectional design, one cannot exclude that emotional distance could be key in maintainingwhile playing no role in instigating — the network system comprising parental burnout, marital 
conflicts, and the associated child maltreatment (see also the discussion below about the distinction between probabilistic dependence and temporal antecedence). There is however no other evidence regarding this hypothesis (either for or against), as so far, most research in parental burnout has viewed and analyzed parental burnout as a unitary construct, utilizing only the total parental burnout score. We thus call on future studies to use not only the global score but to also distinguish between the subscales. This will allow researchers to disentangle the role each of the subscales plays in the onset and maintenance of parental burnout, as well as to identify if the subscales differentially impact related variables, such as child maltreatment or partner conflict.

Distinguishing variables that differentially affect onset and maintenance of parental burnout also would be pivotal in identifying targets ripe for prevention and intervention. Emotional exhaustion and the associated chronic lack of resources, if implicated in the onset of parental burnout, would be important to target for prophylactic efforts. Emotional distance, if central in the maintenance of parental burnout as this study suggests, would be crucial in the development of treatments. Indeed, network theory suggests that "turning off" a highly central node, such as by down-regulating a variable through treatment, should decrease activation throughout the network (McNally, 2016). If this holds true for the models investigated in this study, a treatment that specifically decreases the amount of emotional distance a parent feels toward their child(ren) should also lead to downstream improvement not only in the other parental burnout symptoms but also in child-maltreatment and partner-conflict variables. An example of attempting to specifically reduce emotional distance could include mental imagery exercises such as imagery rescripting or positive imagery re-training (Holmes \& Mathews, 2010), where parents would concretely visualize enjoying and valuing moments spent with children (for more clinical recommendations about mental imagery, see also Blackwell, 2019). Similarly, because the network perspective assumes that nodes having high bridge 
centrality may be more likely to propagate activation through the entire network when reactivated after treatment (e.g., Cramer et al., 2010), future research should examine whether ineffective parenting acts as a potential harbinger of relapse deserving careful audit during follow-up. Note that current treatment efforts for parental burnout already aim to do so via work with parents to improve child-rearing practices (Brianda et al., 2020). Before investigating treatment options in more depth, however, future research needs to further investigate the distinct roles of the different parental burnout subscales and confirm this distinction between onset and maintenance variables, using longitudinal data.

Indeed, one of our study's limitations is that it analyses cross-sectional data. The only insight into the direction of associations is from the DAG, which uses probabilistic methods to provide clues about the direction of probabilistic dependence between the variables. However, when considering DAGs, one must not confuse the direction of probabilistic dependence between variables with temporal antecedence (for a discussion, see Pearl, 2009). Indeed, DAGs encode the conditional probability distribution of the variables. In this way, DAGs can be decomposed as a product of the conditional distribution of each node given its parent nodes in the graph, thus rendering DAGs capable of indicating whether the presence of node A probabilistically implies node B more than vice versa (Heeren et al., 2020). However, it does not imply the temporal precedence of node B. Moreover, the DAGs, by definition, assume that the relationships between variables are acyclic (i.e., no feedback loop). On the other hand, it has been suggested that one may easily gauge the extent of the directional flipflopping in the DAG by looking at the thickness of the edge (e.g., Heeren et al., 2020; McNally et al., 2017). Indeed, the thickness of the edge represents the probability that the edge points in the direction depicted but does not imply that the probability of the edge pointing in the other direction is zero (Heeren et al., 2020). Here, several edges were thin. For instance, the edge from inefficacy to violence pointed in its depicted direction in only $51 \%$ of 
the bootstrapped networks, thus indicating that it pointed in the other direction in $49 \%$ of the bootstrapped networks. Likewise, the edge from emotional distance to exhaustion pointed in its current direction in only $53 \%$ of the bootstrapped networks, thus indicating that it pointed in the other direction in $47 \%$ of the bootstrapped networks. The direction of prediction between these variables may thus go both ways. To confirm and further investigate the direction of association between variables, the next step is to move to intensive time-series data which will allow a direct investigation into the temporal relationship between variables, while also assessing possible feedback loops (Bringmann et al., 2013). This would also allow a micro-level assessment of how parental burnout variables interact with each other and with child-maltreatment and partner-conflict variables, thus allowing insight into the mechanisms of parental burnout.

We also did not measure any potentially relevant moderators, such as personality traits, attachment patterns, or psychopathology symptoms, all of which could affect how parental burnout impacts the children or the spouse. However, the number of parameters grows quickly with each additional node (Epskamp et al., 2018), and so for this first network analysis on parental burnout, we chose to include the minimum number of nodes in the network to ensure that we had adequate power (for information on estimating power in network analyses, see the supplementary materials). We hope that future research, however, will investigate how these types of moderating variables could impact the way parental burnout interacts with behavior toward children and toward the spouse.

Some might find it a limitation that this study does not directly compare parents with severe parental burnout to those unaffected; however, we do not view this as a limitation as using an unselected sample allows for the generalizability of the results and protects against a restricted range issue. More crucially, however, there currently are no accepted clinical cutoffs for parental burnout (Roskam et al., 2017, 2018). And although all scales are skewed 
toward lower scores (since our sample is unselected), all variables nonetheless show a wide variability in scores. The only variables whose scores do not span the entire scale are neglect and abuse, which is to be expected since maximum scores on these scales represent frequent and regular abuse toward children (although there are also no clinical cut-offs for which scores on these scales "officially" cross the line into child abuse). Thus, a connection between (for example) emotional distance and neglect represents that higher scores of emotional distance are associated with an increased frequency of neglectful behaviors, but this increased frequency could be from "never" to "once a month."

Finally, another limitation is that parents self-reported all variables. Parents may thus have felt uncomfortable answering some of the items truthfully, particularly items relating to child maltreatment. On the other hand, the only validated method to measure parental burnout is currently with self-reported questionnaires. In any case, it is extremely challenging to collect datasets large enough for network analyses (e.g., minimum hundreds of participants). that include third-party assessments on child maltreatment — or, indeed, on parental burnout. Future research should nonetheless attempt to investigate other forms of data collection, such as through third party observations from other family members or teachers, or through structured interviews with psychologists or other trained professionals.

\section{Conclusion}

Emotional distance emerges as one of the most central variables in the network, driving the activation of the other parental burnout variables, as well as variables measuring child maltreatment and parental conflict. Neglect and violence towards are also highly central and connected to most of the network. In contrast, partner estrangement and partner conflicts are only associated with parental burnout variables through child maltreatment variables, suggesting that problems with the partner arise from child maltreatment. These results, stemming from cross-sectional data, suggest that emotional distance could play a crucial role 
in maintaining parental burnout and the associated child maltreatment, but further research is needed to confirm and further disentangle the mechanisms of parental burnout. 


\section{References}

Barabási, A. L. (2012). The network takeover. Nature Physics, 8(1), 14-16.

https://doi.org/10.1038/nphys2188

Berger, R. P., Fromkin, J. B., Stutz, H., Makoroff, K., Scribano, P. V., Feldman, K., Tu, L. C., \& Fabio, A. (2011). Abusive head trauma during a time of increased unemployment:

A multicenter analysis. Pediatrics, 128(4), 637-643.

https://doi.org/10.1542/peds.2010-2185

Bernstein, E. E., Heeren, A., \& McNally, R. J. (2017). Unpacking Rumination and Executive

Control: A Network Perspective. Clinical Psychological Science, 5(5), 816-826.

https://doi.org/10.1177/2167702617702717

Bernstein, E. E., Heeren, A., \& McNally, R. J. (2019). Reexamining trait rumination as a system of repetitive negative thoughts: A network analysis. Journal of Behavior Therapy and Experimental Psychiatry, 63, 21-27. https://doi.org/10.1016/j.jbtep.2018.12.005

Blackwell, S. E. (2019). Mental imagery: From basic research to clinical practice. Journal of Psychotherapy Integration, 29(3), 235-247. https://doi.org/10.1037/int0000108 Borgatti, S. P. (2005). Centrality and network flow. Social Networks, 27(1), 55-71.

https://doi.org/10.1016/j.socnet.2004.11.008

Blanchard, M. A., \& Heeren, A. (2020). Why we should move from reductionism and embrace a network approach to parental burnout. New Directions for Child and Adolescent Development. Advance online publication. https://doi.org/10.1002/cad.20377

Borsboom, D. (2017). A network theory of mental disorders. World Psychiatry, 16(1), 5-13. https://doi.org/10.1002/wps.20375

Borsboom, D., \& Cramer, O. J. (2013). Network analysis: An integrative approach to the structure of psychopathology. Annual Review of Clinical Psychology, 9, 91-121. https://doi.org/10.1146/annurev-clinpsy-050212-185608 
Brianda, M. E., Roskam, I., Gross, J. J., Franssen, A., Kapala, F., Gérard, F., \& Mikolajczak, M. (2020). Treating parental burnout: Impact of two treatment modalities on burnout symptoms, emotions, hair cortisol, and parental neglect and violence. Psychotherapy and Psychosomatics, 1-3. https://doi.org/10.1159/000506354

Bringmann, L. F., Vissers, N., Wichers, M., Geschwind, N., Kuppens, P., Peeters, F., Borsboom, D., \& Tuerlinckx, F. (2013). A network approach to psychopathology: new insights into clinical longitudinal data. PLoS ONE, 8(4).

https://doi.org/10.1371/journal.pone.0060188

Cheng, H., Wang, W., Wang, S., Li, Y., Liu, X., \& Li, Y. (2020). Validation of a Chinese Version of the Parental Burnout Assessment. Frontiers in Psychology, 11, 321. https://doi.org/10.3389/fpsyg.2020.00321Contreras, A., Nieto, I., Valiente, C., Espinosa, R., \& Vazquez, C. (2019). The study of psychopathology from the network analysis perspective: A systematic review. Psychotherapy and Psychosomatics, 88(2), 71-83. https://doi.org/10.1159/000497425

Costantini, G., Epskamp, S., Borsboom, D., Perugini, M., Mõttus, R., Waldorp, L. J., \& Cramer, A. O. J. (2015). State of the aRt personality research: A tutorial on network analysis of personality data in R. Journal of Research in Personality, 54, 13-29. https://doi.org/10.1016/j.jrp.2014.07.003

Costenbader, E., \& Valente, T. W. (2003). The stability of centrality measures when networks are sampled. Social Networks, 25(4), 283-307. https://doi.org/10.1016/S03788733(03)00012-1

Cramer, A. O. J., Lourens, J. W., Van Der Maas, H. L. J., \& Borsboom, D. (2010). Comorbidity: A network perspective. Behavioral and Brain Sciences, 33, 137-193. https://doi.org/10.1017/S0140525X09991567

Cramer, A. O. J., van der Sluis, S., Noordhof, A., Wichers, M., Geschwind, N., Aggen, S. H., 
Kendler, K. S., \& Borsboom, D. (2012). Dimensions of normal personality as networks in search of equilibrium: You can't like parties if you don't like people. European Journal of Personality, 26(4), 414-431. https://doi.org/10.1002/per.1866

Csardi, G., \& Nepusz, T. (2006). The igraph software package for complex network research. InterJournal Complex Systems, 1695.

Curtis, T., Miller, B. C., \& Berry, E. H. (2000). Changes in reports and incidence of child abuse following natural disasters. Child Abuse \& Neglect, 24(9), 1151-1162. https://doi.org/10.1016/S0145-2134(00)00176-9

Demerouti, E., Nachreiner, F., Bakker, A. B., \& Schaufeli, W. B. (2001). The job demandsresources model of burnout. Journal of Applied Psychology, 86(3), 499-512. https://doi.org/10.1037/0021-9010.86.3.499

Elliott, H., Jones, P. J., \& Schmidt, U. (2019). Central symptoms predict post-treatment outcomes and clinical impairment in anorexia nervosa: A network analysis. Clinical Psychological Science. https://doi.org/10.1177/2167702619865958

Epskamp, S., Borsboom, D., \& Fried, E. I. (2018). Estimating psychological networks and their accuracy: A tutorial paper. Behavior Research Methods, 50(1), 195-212. https://doi.org/10.3758/s13428-017-0862-1

Epskamp, S., Cramer, A. O. J., Waldorp, L. J., Schmittmann, V. D., \& Borsboom, D. (2012). qgraph: Network visualizations of relationships in psychometric data. Journal of Statistical Software, 48(4), 1-18. https://doi.org/10.18637/jss.v048.i04

Epskamp, S., \& Fried, E. I. (2018). A tutorial on regularized partial correlation networks. Psychological Methods, 23(4), 617-634. https://doi.org/10.1037/met0000167

Everaert, J., \& Joormann, J. (2019). Emotion regulation difficulties related to depression and anxiety: A network approach to model relations among symptoms, positive reappraisal, and repetitive negative thinking. Clinical Psychological Science, 7(6), 1304-1318. 
https://doi.org/10.1177/2167702619859342

Foygel, R., \& Drton, M. (2011). Bayesian model choice and information criteria in sparse generalized linear models. arXiv. https://doi.org/10.1038/srep05918. https://arxiv.org/abs/1112.5635

Fried, E. I. (2016, November 26). New paper: Differential variability of variables drives network structure. Psych Networks. https://psych-networks.com/new-paper-differentialvariability-items-drives-network-structure/

Friedman, J., Hastie, T., \& Tibshirani, R. (2011). glasso: Graphical lasso-estimation of Gaussian graphical models (R package version 1.7).

Griffith, A. K. (2020). Parental burnout and child maltreatment during the COVID-19 pandemic. Journal of Family Violence. https://doi.org/10.1007/s10896-020-00172-2

Halbesleben, J. R. B., \& Bowler, W. M. (2007). Emotional exhaustion and job performance: The mediating role of motivation. Journal of Applied Psychology, 92(1), 93-106. https://doi.org/10.1037/0021-9010.92.1.93

Hansotte, L., Nguyen, N., Roskam, I., Stinghlamber, F., \& Mikolajczak, M. (2019). Are all burned out parents neglectful and violent? A latent profile. [Manuscript submitted for publication]. Psychological Sciences Research Institute, UCLouvain.

Heeren, A., Bernstein, E.E., \& McNally, R.J. (2020). Bridging maladaptive social self-beliefs and social anxiety: A network perspective. Journal of Anxiety disorders, 74, 102267. https://doi.org/10.1016/j.janxdis.2020.102267

Heeren, A., Jones, P. J., \& McNally, R. J. (2018). Mapping network connectivity among symptoms of social anxiety and comorbid depression in people with social anxiety disorder. Journal of Affective Disorders, 228, 75-82.

https://doi.org/10.1016/j.jad.2017.12.003

Heeren, A., \& McNally, R. J. (2018). Social anxiety disorder as a densely interconnected 
network of fear and avoidance for social situations. Cognitive Therapy and Research, 42, 103-113. https://doi.org/10.1007/s10608-017-9876-3

Hittner, J. B., May, K., \& Silver, N. C. (2003). A Monte Carlo evaluation of tests for comparing dependent correlations. Journal of General Psychology, 130(2), 149-168. https://doi.org/10.1080/00221300309601282

Holmes, E. A., \& Mathews, A. (2010). Mental imagery in emotion and emotional disorders. Clinical Psychology Review, 30, 349-362. https://doi.org/10.1016/j.cpr.2010.01.001

Jiang, H., Fei, X., Liu, H., Roeder, K., Lafferty, J., Wasserman, L., Li, X., \& Zhao, T. (2019). huge: High-dimensional undirected graph estimation (R package version 1.3.2).

Jones, P. J. (2018). Networktools: Tools for identifying important nodes in networks (R Package Version 1.1.1.).

Jones, P. J., Ma, R., \& McNally, R. J. (2019). Bridge centrality: A network approach to understanding comorbidity. Multivariate Behavioral Research, 1-15. https://doi.org/10.1080/00273171.2019.1614898

Lee, R. T., \& Ashforth, B. E. (1993). A longitudinal study of burn-out among supervisors and managers: Comparisons between the Leiter and Maslach (1988) and Golembiewski et al. (1986) models. Organizational Behavior \& Human Decision Processes, 54(3), 369-398.

Leiter, M. P. (1993). Burnout as a developmental process: Consideration of models. In W. Schaufeli, C. Maslach, \& T. Marek (Eds.), Professional burnout: Recent developments in theory and research. Taylor \& Francis.

Leiter, M. P., \& Maslach, C. (1988). The impact of interpersonal environment on burnout and organizational commitment. Journal of Organizational Behavior, 9, 297-308. https://doi.org/10.1002/job.4030090402

Lindström, C., Åman, J., \& Norberg, A. L. (2011). Parental burnout in relation to sociodemographic, psychosocial and personality factors as well as disease duration 
and glycaemic control in children with Type 1 diabetes mellitus. Acta Paediatrica, 100(7), 1011-1017. https://doi.org/10.1111/j.1651-2227.2011.02198.x

Maslach, C., Jackson, S. E., \& Leiter, M. P. (2010). Maslach Burnout Inventory Manual. Mind Garden.

McNally, R. J. (2016). Can network analysis transform psychopathology? Behaviour Research and Therapy, 86, 95-104. https://doi.org/10.1016/j.brat.2016.06.006

McNally, R. J., Heeren, A., \& Robinaugh, D. J. (2017). A Bayesian network analysis of posttraumatic stress disorder symptoms in adults reporting childhood sexual abuse. European Journal of Psychotraumatology, 8(sup3), 1341276. https://doi.org/10.1080/20008198.2017.1341276

Mikolajczak, M., Brianda, M. E., Avalosse, H., \& Roskam, I. (2018). Consequences of parental burnout: Its specific effect on child neglect and violence. Child Abuse and Neglect, 80, 134-145. https://doi.org/10.1016/j.chiabu.2018.03.025

Mikolajczak, M., Gross, J. J., \& Roskam, I. (2019). Parental burnout: What is it, and why does it matter? Clinical Psychological Science, 7(6), 1319-1329. https://doi.org/10.1177/2167702619858430

Mikolajczak, M., Gross, J. J., Stinglhamber, F., Lindahl Norberg, A., \& Roskam, I. (2020). Is Parental Burnout distinct from job burnout and depressive symptoms? Clinical Psychological Science, 8(4), 673-689. https://doi.org/10.1177/2167702620917447

Mikolajczak, M., Raes, M. E., Avalosse, H., \& Roskam, I. (2018). Exhausted parents: Sociodemographic, child-related, parent-related, parenting and family-functioning correlates of parental burnout. Journal of Child and Family Studies, 27(2), 602-614. https://doi.org/10.1007/s10826-017-0892-4

Mikolajczak, M., \& Roskam, I. (2018). A theoretical and clinical framework for parental burnout: The balance between risks and resources (BR2). Frontiers in Psychology, 9, 
886. https://doi.org/10.3389/fpsyg.2018.00886

Papini, S., Rubin, M., Telch, M. J., Smits, J., \& Hien, D. A. (2020). Pretreatment posttraumatic stress disorder symptom network metrics predict the strength of the association between node change and network change during treatment. Journal of Traumatic Stress, 33, 64-71. https://doi.org/10.1002/jts.22379

Pearl, J. (2009). Causality: Models, reasoning and inference. Cambridge, MA, USA: Cambridge University Press

Pearl, J., Glymour, M., \& Jewell, N. P. (2016). Causal inference in statistics: A primer. John Wiley \& Sons.

Pines, A., \& Aronson, E. (1988). Career burnout: Causes and cures. Free press.

Reichardt, J., \& Bornholdt, S. (2006). Statistical mechanics of community detection. Physical Review E - Statistical, Nonlinear, and Soft Matter Physics, 74(1), 1-14. https://doi.org/10.1103/PhysRevE.74.016110

Robinaugh, D. J., Hoekstra, R. H. A., Toner, E. R., \& Borsboom, D. (2019). The network approach to psychopathology : a review of the literature $2008-2018$ and an agenda for future research. Psychological Medicine, 50(3), 1-14. https://doi.org/https:// doi.org/10.1017/S0033291719003404

Robinaugh, D. J., LeBlanc, N. J., Vuletich, H. A., \& McNally, R. J. (2014). Network analysis of persistent complex bereavement disorder in conjugally bereaved adults. Journal of Abnormal Psychology, 123(3), 510-522. https://doi.org/doi:10.1037/abn0000002

Robinaugh, D. J., Millner, A. J., \& McNally, R. J. (2016). Identifying highly influential nodes in the complicated grief network. Journal of Abnormal Psychology, 125(6), 747-757. https://doi.org/10.1037/abn0000181

Roskam, I., Brianda, M. E., \& Mikolajczak, M. (2018). A step forward in the conceptualization and measurement of parental burnout: The Parental Burnout 
Assessment (PBA). Frontiers in Psychology, 9, 758.

https://doi.org/10.3389/fpsyg.2018.00758

Roskam, I., Raes, M. E., \& Mikolajczak, M. (2017). Exhausted parents: Development and preliminary validation of the parental burnout inventory. Frontiers in Psychology, 8, 163. https://doi.org/10.3389/fpsyg.2017.00163

Scutari, M. (2010). Learning Bayesian networks with the bnlearn R Package. Journal of Statistical Software, 35(3), 1-22. https://doi.org/10.18637/jss.v035.i03

Scutari, M., \& Denis, J. (2015). Bayesian networks: With examples in R. CRC Press: Boca Raton, FL.

Scutari, M., \& Nagarajan, R. (2013). Identifying significant edges in graphical models of molecular networks. Artificial Intelligence in Medicine, 57, 207-217.

Sorkkila, M., \& Aunola, K. (2020). Risk factors for Parental Burnout among Finnish Parents: The role of socially prescribed perfectionism. Journal of Child and Family Studies, 29, 648-659. https://doi.org/10.1007/s10826-019-01607-1

Traag, V. A., \& Bruggeman, J. (2009). Community detection in networks with positive and negative links. Physical Review E - Statistical, Nonlinear, and Soft Matter Physics, 80(3), 1-6. https://doi.org/10.1103/PhysRevE.80.036115

Terluin, B., de Boer, M. R., \& de Vet, H. C. W. (2016). Differences in connection strength between mental symptoms might be explained by differences in variance: Reanalysis of network data did not confirm staging. PloS ONE, 11(11), e0155205.

van Borkulo, C. D., Boschloo, L., Kossakowski, J. J., Tio, P., Schoevers, R. A., Borsboom, D., \& Waldorp, L. J. (2017). Comparing network structures on three aspects: A permutation test. ResearchGate. https://doi.org/10.13140/RG.2.2.29455.38569

Weston, S. J., Ritchie, S. J., Rohrer, J. M., \& Przybylski, A. K. (2019). Recommendations for increasing the transparency of analysis of preexisting data sets. Advances in Methods and 
Practices in Psychological Science, 2(3), 214-227.

https://doi.org/10.1177/2515245919848684

Yang, Z., Algesheimer, R., \& Tessone, C. J. (2016). A comparative analysis of community detection algorithms on artificial networks. Scientific Reports, 6, 30750.

https://doi.org/10.1038/srep30750 


\section{Table 1}

Mean, Standard Deviation (SD), and Range for Sample Characteristics

\begin{tabular}{lccc}
\hline Demographic Variable & Mean & SD & Range \\
\hline Age (Women) & 38.23 & 7.28 & $23-63$ \\
Age (Men) & 42.61 & 8.47 & $27-66$ \\
Number of Children & 2.30 & 1.38 & $1-7$ \\
Age of Children & 8.49 & 6.70 & $0-35$ \\
\hline
\end{tabular}




\section{Table 2}

Mean, Standard Deviation (SD), Minimum (Min), Maximum (Max), Skewness, and Kurtosis of Each Variable (Before Nonparanormal Transformation)

\begin{tabular}{lcccccc}
\hline Variable & Mean & SD & Min & Max & Skewness & Kurtosis \\
\hline Exhaustion & 14.97 & 11.39 & 0 & 48 & 0.87 & -0.05 \\
Distance & 9.34 & 8.51 & 0 & 47 & 1.47 & 2.23 \\
Inefficacy & 8.23 & 6.90 & 0 & 36 & 1.24 & 1.40 \\
Neglect & 26.91 & 9.17 & 17 & 84 & 2.00 & 5.91 \\
Violence & 22.75 & 7.58 & 15 & 81 & 2.57 & 11.19 \\
Partner Estrangement & 7.81 & 3.76 & 5 & 35 & 2.50 & 8.38 \\
Partner Conflict & 5.38 & 2.50 & 2 & 14 & 0.97 & 0.35 \\
\hline
\end{tabular}

Note . Exhaustion $=$ Emotional exhaustion; Distance $=$ Emotional distance; Inefficacy $=$ Loss of parental accomplishment and efficacy; Neglect $=$ Neglectful behaviors toward children; Violence $=$ Violent behaviors toward children 


\section{Figure 1}

Pearson product-moment correlations between each nonparanormal-transformed variable

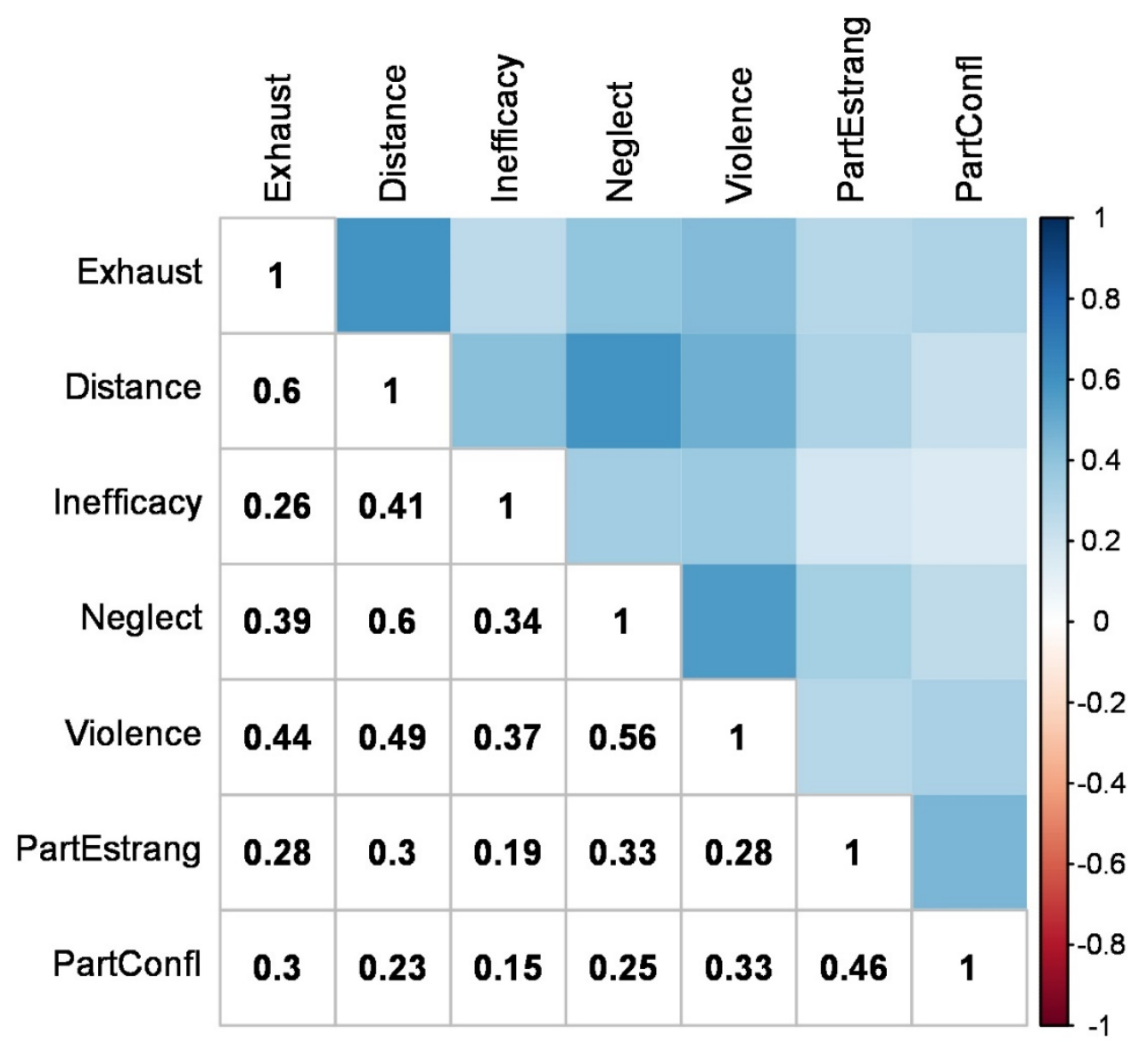

Note . Exhaust $=$ Emotional exhaustion; Distance $=$ Emotional distance; Inefficacy $=$ Loss of parental accomplishment and efficacy; Neglect $=$ Neglectful behaviors toward children;

Violence $=$ Violent behaviors toward children; PartEstrang $=$ Partner estrangement; PartConfl

$=$ Conflicts with partner. 


\section{Figure 2}

Graphical Gaussian Model Network Constructed via the Graphical LASSO

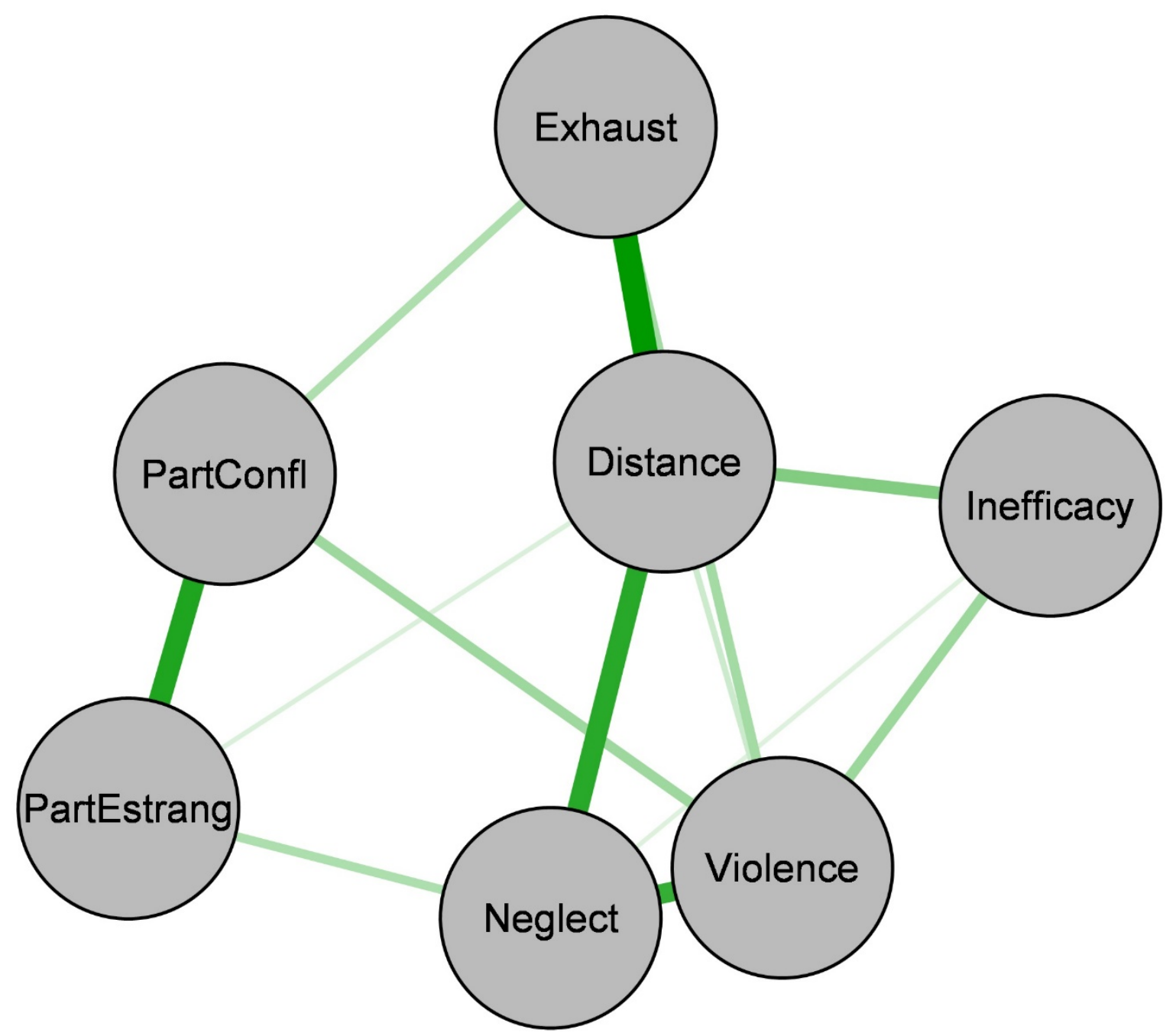

Note. An edge's thickness represents the magnitude of the association between the two relevant nodes (with the thickest edge representing a value of ....). Green lines represent positive regularized partial correlations. Exhaust $=$ Emotional exhaustion; Distance $=$ Emotional distance; Inefficacy $=$ Loss of parental accomplishment and efficacy; Neglect $=$ Neglectful behaviors toward children; Violence $=$ Violent behaviors toward children; PartEstrang $=$ Partner estrangement; PartConfl $=$ Conflicts with partner. 


\section{Figure 3}

Expected Influence and Bridge Expected Influence Estimates of the Graphical LASSO

A

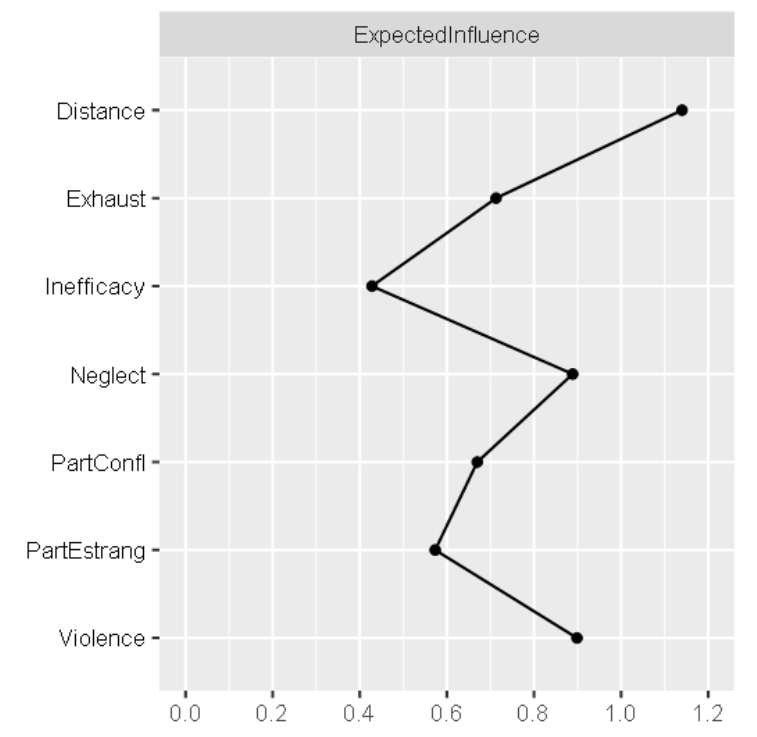

B

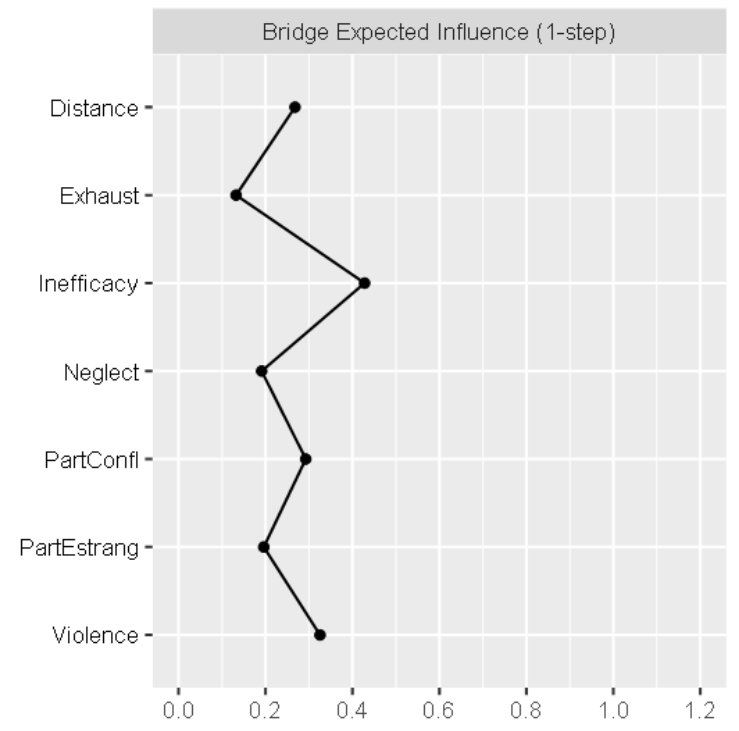

Note . Exhaust $=$ Emotional exhaustion; Distance $=$ Emotional distance; Inefficacy $=$ Loss of parental accomplishment and efficacy; Neglect = Neglectful behaviors toward children;

Violence $=$ Violent behaviors toward children; PartEstrang $=$ Partner estrangement; PartConfl $=$ Conflicts with partner. 


\section{Figure 4}

Directed Acyclic Graphs (DAGs)
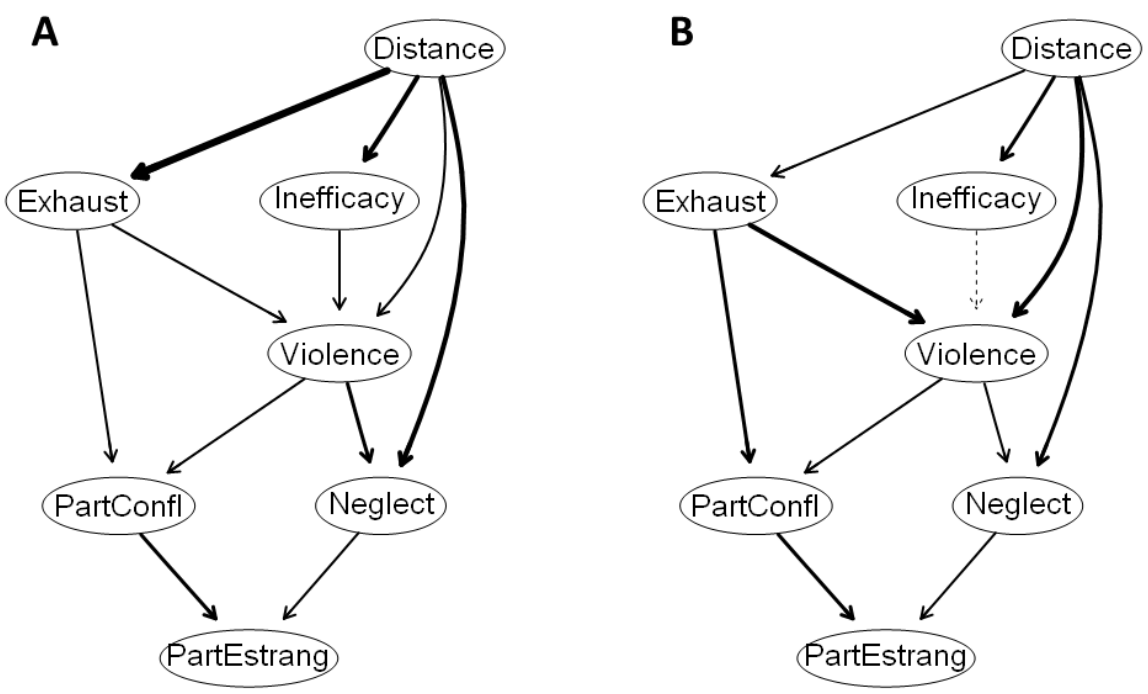

Note. Panel A: Presence of edges: Edge thickness indicates the importance of that edge to the overall network structure, with greater thickness signifying that an edge is more crucial to model fit. Thickness reflects the change in the Bayesian Information Criterion of the model when that edge is removed. For this graph, solid lines represent that the present of an edge improves model fit (a dashed line would represent an edge whose presence worsens model fit). Panel B: Direction of edges: edge thickness indicates directional probability, or in what percentage of the fitted networks the edge went in that direction. Edge thickness is drawn proportionately, such that a thicker arrow indicates a higher directional probability. For this graph, a solid line represents that an edge was present in its current direction in at least $51 \%$ of the 10,000 bootstrapped networks, while a dotted line represents an edge present in its current direction in less than 51\%. For both Panels A and B, exact edge weights can be found in Table S1 in the supplementary materials. Exhaust = Emotional exhaustion; Distance = Emotional distance; Inefficacy $=$ Loss of parental accomplishment and efficacy; Neglect = Neglectful behaviors toward children; Violence $=$ Violent behaviors toward children; PartEstrang $=$ Partner estrangement; PartConfl $=$ Conflicts with partner. 